\title{
Rate and predictors of nodal pathological complete response following neoadjuvant endocrine treatment in clinically biopsy-proven node- positive breast cancer patients
}

\author{
Robert-Jan Schipper ${ }^{a}$, , Anne de Bruijn ${ }^{\text {a }}$, Adri C. Voogd ${ }^{\text {b, c }}$, Johanne G. Bloemen ${ }^{\text {a }}$, \\ Yvonne E. Van Riet ${ }^{a}$, Birgit E.P. Vriens ${ }^{\mathrm{d}}$, Marjolein L. Smidt ${ }^{\text {c, e }}$, Sabine Siesling ${ }^{\text {b, f }}$, \\ Maurice J.C. van der Sangen ${ }^{g}$, Grard A.P. Nieuwenhuijzen ${ }^{a}$ \\ a Department of Surgery, Catharina Hospital Eindhoven, the Netherlands \\ b Department of Research and Development, Netherlands Comprehensive Cancer Organization (IKNL), Utrecht, the Netherlands \\ ${ }^{\text {c } G R O W-S c h o o l ~ f o r ~ O n c o l o g y ~ a n d ~ D e v e l o p m e n t a l ~ B i o l o g y, ~ M a a s t r i c h t ~ U n i v e r s i t y ~ M e d i c a l ~ C e n t e r, ~ M a a s t r i c h t, ~ t h e ~ N e t h e r l a n d s ~}$ \\ ${ }^{\mathrm{d}}$ Department of Internal Medicine, Catharina Hospital Eindhoven, the Netherlands \\ e Department of Surgery, Maastricht University Medical Center +, Maastricht, the Netherlands \\ ${ }^{\mathrm{f}}$ Department of Health Technology and Services Research, Technical Medical Centre, University of Twente, Enschede, the Netherlands \\ ${ }^{g}$ Department of Radiotherapy, Catharina Hospital Eindhoven, the Netherlands
}

\section{A R T I C L E I N F O}

\section{Article history:}

Received 16 February 2021

Received in revised form

10 April 2021

Accepted 28 April 2021

Available online 18 May 2021

\section{Keywords:}

Breast cancer

Neoadjuvant endocrine therapy

Clinical node-positive

Axillary complete response

\begin{abstract}
A B S T R A C T
Background: Data on effectiveness and optimal use of neoadjuvant endocrine therapy (NET) in clinically biopsy-proven node-positive breast cancer is lacking. This study examined the incidence of axillary pathological complete response (pCR) on NET in clinically biopsy-proven node-positive breast cancer patients. Secondary, patient and tumour characteristics, as well as the optimal duration of NET in relation to the occurrence of axillary pCR were investigated.

Material and methods: Patients diagnosed with primary hormone receptor positive, HER2 negative breast cancer between 2014 and 2019, with at least one positive axillary lymph node (pathologically proven), treated with NET were selected from the Netherlands Cancer Registry. The incidence of axillary pCR in combination with patient, tumour and treatment characteristics was analysed.

Results: In a population of 561 patients, an axillary pCR of $7.3 \%$ on NET was observed. Median length of treatment was 8.1 months in the patients without vs. 8.8 months in those with axillary pCR, with no statistically significant difference. A p-value $<0.30$ was found for age, histologic type, clinical tumour status, hormone receptor status and the type of NET in univariable analysis. After multivariable logistic regression analyses, none of these variables were independently associated with the likelihood of an axillary pCR.

Conclusion: The rate of axillary pCR after NET in HR + HER2-clinically biopsy-proven node-positive breast cancer patients is low. Factors independently associated with the likelihood of an axillary pCR could not be identified. More research is warranted regarding optimizing the duration of NET and the prognostic value of residual disease in the axilla after NET.
\end{abstract}

๑ 2021 Elsevier Ltd, BASO The Association for Cancer Surgery, and the European Society of Surgical Oncology. All rights reserved.

\section{Introduction}

Historically, primary endocrine treatment (PET) is used in patients with hormone receptor positive $(\mathrm{HR}+)$ breast cancer who are

\footnotetext{
* Corresponding author.

E-mail address: info@rjschipper.nl (R.-J. Schipper).
}

considered too frail for surgery. In addition, neoadjuvant endocrine treatment (NET) is used in patients with locally advanced HR + human epidermal growth factor receptor-2 negative (HER2-) breast cancer, who are unfit for neoadjuvant chemotherapy (NAC), but fit for surgery, to facilitate breast conserving surgery (BCS). The threshold to administer chemotherapy in HR + HER2-breast cancer is rising, mainly as a result of the introduction of genetic profiling [1-3]. In patients with HR + HER2-tumours with clinically node- 
positive breast cancer and a favourable genetic profile, chemotherapy can be safely avoided [4]. This development makes NET an attractive alternative approach for a substantial number of patients. Nonetheless, recent data show that its use continues to be marginal, with only $2.3 \%$ of patients with HR + stage II and III breast cancer receiving NET [5]. It is known that 3-4 months of NET enables the use of BCS in 50\% of the patients in whom a mastectomy was considered first, at the cost of a progression rate of $6.5 \%$ of the primary tumour during NET [6]. By extending treatment duration up to 12 months, BCS becomes feasible in up to $87.5 \%$ of the patients at the cost of $10 \%$ of these patients, who experience local progression [6]. In line with these figures, also pCR rates of the breast tumour also rise from $2.5 \%$ after 4 months of treatment up to $17.5 \%$ after 12 months of treatment [6].

Apart from the focus of NET on reducing the size of the primary tumour to facilitate the use of BCS, there could be a role of NET in minimizing the extent of axillary surgery. The ongoing trend of minimizing the extent of axillary surgery in primary clinically node-positive breast cancer patients has been investigated for NAC in many trials studying alternative surgical approaches of the axillary lymph nodes after NAC. Such approaches are sentinel lymph node biopsy (SLNB) after NAC $[7,8]$, the performance of the Marking the Axilla with a Radioactive Iodine seed (MARI) procedure [9], a target axillary dissection (TAD) [10] or a combination of multiple techniques [11]. All these studies resulted in omitting an axillary lymph node dissection (ALND) in many patients receiving NAC, but mainly in those with triple negative and HER2 positive breast cancers. In patients with HR + HER2-tumours the incidence of axillary pCR after NAC is reported to be around $7.4-15 \%$, thereby leaving little room for the omittance of an ALND [12-14].

In NET fewer trials on omitting extensive surgery have been performed. Even so, a recent systematic review of the literature showed that the incidence of axillary PCR after NET is around 10-14.5\%, which seems to be slightly higher than in NAC [15]. However, in this review no information was available on the duration of NET. Another publication based on data from the National Cancer Database (NCDB, United States of America) reported that patients with clinically node-positive ( $\mathrm{pN} 1$ ) breast cancer undergoing NET instead of NAC were less likely to undergo an ALND [5]. Probably, selection bias has accounted for these lower odds. Hence, the optimal management of patients with clinically nodepositive, HR + HER2-breast cancer treated with NET is unknown.

Therefore, in this study we examined the incidence of axillary pCR on NET in clinically biopsy-proven node-positive breast cancer patients. Secondly, patient and tumour characteristics, as well as the optimal duration of NET in relation to the occurrence of axillary pCR were investigated.

\section{Material and methods}

\section{Patients}

Data were obtained from the Netherlands Cancer Registry (NCR), which is managed by the Netherlands Comprehensive Cancer Organization (IKNL). The NCR collects data of all patients diagnosed with breast cancer in the Netherlands. Trained data collection registrars extract data from patients' records. Since this is a retrospective, anonymous study, there was no need for informed consent. For this study, all patients diagnosed with primary invasive breast cancer of no specific type (NST) or lobular breast cancer in the period between 2014 and 2019, with at least one positive axillary lymph node (pathological proven by ultrasound guided fine needle aspiration cytology or core biopsy), with a HR + HER2-status and treated by NET were included. Patients receiving NET for less than 4 weeks and male patients were excluded. Until 2017, TNM/
AJCC tumour staging 7th edition was used and from 2018, the 8th edition.

\section{Treatment}

Patients were treated according to the Dutch breast cancer guidelines. In short, regarding endocrine treatment regimens, premenopausal patients were treated with tamoxifen $20 \mathrm{mg}$ once daily in combination with a GnRH analogue. Postmenopausal patients were treated either with tamoxifen $20 \mathrm{mg}$ once daily or an aromatases inhibitor. The guideline did not provide any suggestions for duration of NET, nor for frequency and technique of choice for treatment evaluation during NET. Type and extent of breast and axillary surgery was based on the preference of the treating physician and/or patient. Four different axillary surgical strategies were used after NET: 1. ALND 2. SLNB. 3 MARI. 4. Combination of both SLNB and MARI.

\section{Pathology}

Core biopsies and surgical specimens were evaluated according to the Dutch breast cancer guidelines [16]. The initial core biopsy of the primary tumour was evaluated using standard haematoxylin and eosin staining, immunohistochemistry and/or fluorescence or chromogenic in situ hybridization for histological subtype, oestrogen and progesterone receptor status (positive if $\geq 10 \%$ ), and HER2 status. In this study, pCR of the breast was defined as the absence of in situ and invasive tumour cells in the resection specimen. Axillary PCR was defined as no or only isolated tumour cells in one or more axillary lymph nodes after NET. Hence, residual micrometastasis were regarded as no axillary pCR.

\section{Statistical analyses}

Descriptive analyses, using chi-square crosstabs, were used to report on the incidence of axillary $\mathrm{PCR}$ in combination with different patient, tumour and treatment characteristics.

To perform univariable and multivariable analyses, different variables were constructed from the NCR. Patients were divided into three age groups $(<50,50-75$, and $>75$ years), and were classified as pre- or post-menopausal. Peri-menopausal patients were regarded as pre-menopausal. Tumours were graded according to the Bloom and Richardson scoring system. In order to investigate the role of the duration of NET on axillary pCR, patients were divided into three groups; $29-180$ days, $181-270$ days and $>270$ days. Univariable and multivariable logistic regression analyses were performed to determine the association between the covariates and the probability to achieve axillary pCR. For the multivariable analyses, stepwise regression with backwards selection was used to identify the covariates that were independently associated with $\mathrm{pCR}$ of variables. Only the covariates with p-value $<0.30$ in the univariable analysis were included in multivariable regression equation. Data analyses were performed using SPSS version 25 (SPSS Inc., Chicago, IL, USA).

\section{Results}

\section{Patients}

A total of 561 patients were identified with HR + HER2-clinical nodal positive breast cancer who received NET followed by surgery between 2014 and 2019. Table 1 shows the clinicopathologic features of the patients included. Median age at diagnosis was 69 years (range 25-92) and mean age was 65.6 years Seventy-eight percent of the patients were postmenopausal and $47 \%$ had a cT2 tumour. All 
Table 1

Patient characteristic of breast cancer patients diagnosed between 2014 and 2019 in the Netherlands $(\mathrm{n}=561)$.

\begin{tabular}{|c|c|c|c|c|c|c|}
\hline & & Total $(\mathrm{N})$ & $\%$ & Axillary pCR (N) & $\%$ & P-value \\
\hline \multirow[t]{4}{*}{ Age years } & & & & & & 0.14 \\
\hline & $<50$ & 92 & 16.4 & 6 & 6.5 & \\
\hline & $50-75$ & 320 & 57.0 & 29 & 9.1 & \\
\hline & $>75$ & 149 & 26.6 & 6 & 4.0 & \\
\hline \multirow[t]{4}{*}{ Menopausal status } & & & & & & 0.44 \\
\hline & Pre & 111 & 19.8 & 6 & 5.4 & \\
\hline & Post & 440 & 78.4 & 33 & 7.5 & \\
\hline & Missing & 10 & 1.8 & 2 & 20.0 & \\
\hline \multirow[t]{3}{*}{ Histologic subtype } & & & & & & 0.14 \\
\hline & NST & 475 & 84.7 & 38 & 8.0 & \\
\hline & Lobular & 86 & 15.3 & 3 & 3.5 & \\
\hline \multirow[t]{5}{*}{ Clinical tumor status } & & & & & & 0.27 \\
\hline & cT1 & 126 & 22.5 & 13 & 10.3 & \\
\hline & cT2 & 266 & 47.4 & 19 & 7.1 & \\
\hline & cT3 & 96 & 17.1 & 7 & 7.3 & \\
\hline & cT4 & 73 & 13.0 & 2 & 2.7 & \\
\hline \multirow[t]{3}{*}{ Clinical nodal status } & & & & & & 0.74 \\
\hline & $\mathrm{cN} 1$ & 539 & 96.1 & 39 & 7.2 & \\
\hline & $\mathrm{cN} 2$ & 22 & 3.9 & 2 & 9.1 & \\
\hline \multirow[t]{4}{*}{ Multifocality } & & & & & & 0.86 \\
\hline & Unifocal & 408 & 72.7 & 31 & 7.6 & \\
\hline & Multifocal & 151 & 26.9 & 10 & 6.6 & \\
\hline & Missing & 2 & 0.4 & 0 & 0.0 & \\
\hline \multirow[t]{4}{*}{ Receptor status } & & & & & & 0.15 \\
\hline & $\mathrm{ER}+\mathrm{PR}+\mathrm{HER} 2-$ & 454 & 80.9 & 29 & 6.4 & \\
\hline & ER + PR - HER2 - & 106 & 18.9 & 11 & 10.4 & \\
\hline & Missing & 1 & 0.2 & 1 & 100 & \\
\hline \multirow[t]{4}{*}{ Grade } & & & & & & 0.52 \\
\hline & 1 & 85 & 15.2 & 7 & 8.2 & \\
\hline & 2 & 329 & 58.6 & 20 & 6.1 & \\
\hline & 3 & 74 & 13.2 & 7 & 9.5 & \\
\hline Missing & & 73 & 13.0 & 7 & 9.6 & \\
\hline \multirow[t]{4}{*}{ DCIS } & & & & & & 0.66 \\
\hline & Not present & 337 & 60.1 & 27 & 8.0 & \\
\hline & Present & 201 & 35.8 & 14 & 7.0 & \\
\hline & Missing & 3 & 0.5 & - & & \\
\hline \multirow[t]{4}{*}{ Screen detected } & & & & & & 0.86 \\
\hline & No & 441 & 78.6 & 33 & 7.5 & \\
\hline & Yes & 100 & 17.8 & 8 & 8.0 & \\
\hline & Missing & 20 & 3.6 & - & 0.0 & \\
\hline \multirow[t]{3}{*}{ Type NET } & & & & & & 0.18 \\
\hline & Tamoxifen $(+/-G n R H)$ & 205 & 36.5 & 19 & 9.3 & \\
\hline & Aromatase inhibitor & 356 & 63.5 & 22 & 6.2 & \\
\hline \multirow[t]{4}{*}{ Duration NET days } & & & & & & 0.82 \\
\hline & $29-180$ & 102 & 18.2 & 6 & 5.9 & \\
\hline & $181-270$ & 280 & 49.9 & 21 & 7.5 & \\
\hline & $>270$ & 179 & 31.9 & 14 & 7.8 & \\
\hline
\end{tabular}

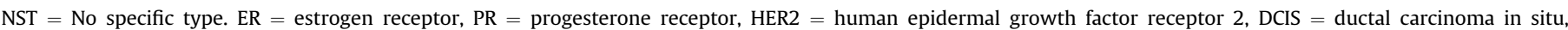
$\mathrm{NET}=$ neoadjuvant endocrine therapy.

tumours were ER positive, and $81 \%$ were also PR positive. Clinical nodal status was $\mathrm{cN} 1$ in $96.1 \%$ and $\mathrm{cN} 2$ in $3.9 \%$ of the patients. Multifocality of the breast tumour was present in $26.9 \%$, and a DCIS component in $35.8 \%$ of the patients with an invasive tumour.

\section{Treatment results}

Tamoxifen was administered in 205 (37\%) patients and an aromatase inhibitor in 356 (63\%) patients. The median duration of NET was 8.0 months with a range of 1-35 months. In 263 patients (46.8\%) BCS was performed. Of all patients, 173 (30.8\%) underwent an immediate ALND. An SLNB was performed in 139 (24.8\%) patients and a MARI procedure in 141 (25.1\%) patients. In 108 (19.3\%) patients a combination of MARI and SLNB was performed: in 50 (46.3\%) of these patients both the MARI procedure and the SLNB showed residual disease, while in 33 (30.5\%) patients the MARI was positive and SLNB was negative. The combination of a positive SLNB and negative MARI occurred in 14 (13.0\%) patients. In $11(10.2 \%)$ patients both MARI and SLNB were negative. A completion ALND was performed in 47 out of the 388 (12.1\%) patients.

Breast and axillary $p C R$

Axillary pCR occurred in 41 patients (7.3\%), while breast pCR

Table 2

ypT status with corresponding ypN status.

\begin{tabular}{llllll}
\hline & ypN0 & ypN1 & ypN2 & ypN3 & Total \\
\hline ypT0 & $14(46.7 \%)$ & $11(33.7 \%)$ & $5(19.6 \%)$ & - & 30 \\
ypT1 & $25(10.4 \%)$ & $189(78.4 \%)$ & $19(7.9 \%)$ & $8(3.3 \%)$ & 241 \\
ypT2 & $2(0.9 \%)$ & $143(64.7 \%)$ & $51(23.1 \%)$ & $25(11.3 \%)$ & 221 \\
ypT3 & - & $25(55.6 \%)$ & $14(31.1 \%)$ & $6(13.3 \%)$ & 45 \\
ypT4 & - & $11(45.8 \%)$ & $10(41.7 \%)$ & $3(12.5 \%)$ & 24 \\
Total & $41(7.3 \%)$ & $379(67.6 \%)$ & $99(17.6 \%)$ & $42(7.5 \%)$ & $\mathbf{5 6 1}$ \\
\hline
\end{tabular}


occurred in 30 of the 651 patients (5.4\%). In only $2.5 \%$ patients (14/ 561 ) both breast and axillary pCR were achieved (Table 2). Residual ypN1, ypN2 and ypN3 were diagnosed in $67.6 \%, 17.6$ and $7.5 \%$ of the patients, respectively. Patients with $\mathrm{cN} 1$ were upstaged after NET and surgery in $23.6 \%$, with ypN2 in $16.7 \%$ and ypN3 in $6.9 \%$ (Table 3 ). There was no statistically significant difference between the nonaxillary $\mathrm{pCR}$ group versus the axillary $\mathrm{pCR}$ group regarding the duration of NET; 8.1 months vs. 8.8 months, respectively $(p=0.26)$.

\section{Factors associated with axillary $p C R$}

In the univariable analyses, none of the variables showed a statistically significant association with likelihood of an axillary pCR (Table 4). A p-value $<0.30$ was found for age, histologic type, clinical tumour status, receptor status and the type of NET and these variables were included in the multivariable logistic regression model. After stepwise regression with backwards selection none of these variables were independently associated with the likelihood of an axillary pCR. Table 4 shows the odds ratios and the p-values for the variables included in the full model.

\section{Discussion}

This nationwide population-based study reported an axillary pCR of 7.3\% after NET in clinically node-positive HR +, HER2 - breast cancer patients in a population of 561 patients. Whether this rate is comparable to the axillary pCR rate of $7.4-15 \%$ after NAC in this subgroup of breast cancer patients remains unknown. This rate after NAC depends mainly on the PR status and tumour grade [12-14]. Selection bias will definitely play a role in whether a patient received NET rather than NAC. Therefore, a head to head comparison is impossible.

Nonetheless, the rate of $7.3 \%$ as reported in the current study is lower than to the axillary pCR rate of $10 \%$ after NET reported by Stafford based on data from the National Cancer Database (NCDB) [15]. An explanation can be found in a different definition of a clinical node-positive patient. In the current study, all clinical nodepositive patients were biopsy-proven, which was not mandatory in the NCDB, in which the clinical nodal status at diagnosis was primarily based on physical examination. Probably, a part of the clinical node-positive patients was incorrectly diagnosed as nodepositive, resulting in a higher axillary pCR rate.

In the current study we were unable to identify factors that were independently associated with axillary pCR. In contrast, Stafford et al. reported a better axillary response in poorly differentiated tumours, and in patients with more favourable pretreatment nodal status [15]. We found a similar trend for both factors, but we were unable to demonstrate statistical significance, because of a type II error due to the lower numbers (561) in our study compared to the study by Stafford et al. (4580) and the relatively weak association. In the study by Stafford et al. axillary pCR is reported in $17.3 \%$ of the patients with poorly differentiated tumours compared to $14.7 \%$ in well-differentiated tumours.

Regarding histological subtype, both Stafford et al. and Montagna et al. reported that lobular carcinomas have a lower axillary $\mathrm{pCR}$ rate compared to ductal carcinomas, which is in line with our

Table 3

cN status with corresponding ypN status.

\begin{tabular}{llllll}
\hline & ypN0 & ypN1 & ypN2 & ypN3 & Total \\
\hline cN1 & $39(7.2 \%)$ & $373(69.2 \%)$ & $90(16.7 \%)$ & $37(6.9 \%)$ & 539 \\
cN2 & $2(9.1 \%)$ & $6(27.3 \%)$ & $9(40.9 \%)$ & $5(22.7 \%)$ & 22 \\
Total & $41(7.3 \%)$ & $379(67.6 \%)$ & $99(17.6 \%)$ & $42(7.5 \%)$ & $\mathbf{5 6 1}$ \\
\hline
\end{tabular}

current study $[15,17]$. It is difficult to understand this phenomenon. Montagna et al. already identified this as an unexpected finding by stating that "lobular carcinomas have a common luminal-A phenotype, thereby making them the perfect theoretical candidates to omit NAC and indicate NET" [17,18]. It may be partly explained by the findings of Truin et al., whom reported no difference in levels of ER and PR expression between invasive ductal and lobular carcinomas [19]. Thornton et al. already reported a higher axillary pCR rate in nodal positive lobular carcinomas treated with NAC compared to NET (13.4\% vs $8.1 \%$ ) in their cohort study [20]. Ultimately, genetic profiling should be used to identify patients with node-positive invasive lobular carcinoma who will benefit from NAC instead of NET in order to achieve axillary downstaging. Nowadays evidence is available showing the independent prognostic value of the outcome of these tests in lobular carcinomas [21-23].

Regarding the duration of NET, a median duration of 8 months was reported in the current study, compared to 4.6 months in the study of Montagna et al. [17]. In their study, axillary pCR was reported in 4 of the 38 (11\%) pathologically proven node-positive patients, compared to $7.4 \%$ in the current study. In our study as well as in the study of Montagna et al., a non-statistically significant difference between the duration of NET and the occurrence of PCR was observed ( 8.8 vs. 8.1 and 6.5 vs. 4.0 months, respectively). Although not statistically significant, the figures suggest that longer treatment may results in a better response, in line with previous studies reporting further reduction in size of the breast tumour by prolonging NET [6]. However, it cannot be ignored that with longer duration of NET, there will probably also be more patients with disease progression [6]. At the moment, there is no evidence available regarding the relation between duration of treatment with NET and the risk of axillary progression of the disease.

In patients with an axillary pCR following NAC or NET, an ALND or adjuvant radiotherapy to the lymph nodes appears to be overtreatment, which is currently investigated in the NSABP B-51/RTOG 1304 trial [24]. The optimal adjuvant treatment of $\mathrm{cN}+$ patients with residual disease in the axilla is also a matter of debate, especially in patients with HR + HER2-grade $1-2$ breast cancer. In this subgroup, the occurrence of ypT0/ypNO after NAC is not associated with event-free survival [25]. Whether there is an association between the degree of regression after NET and survival is unclear. Available evidence suggests that outcomes after NET mirror those of upfront surgery in patients with residual isolated tumour cells, micrometastasis or limited macrometastases (1-2 positive nodes). Probably, this subgroup of tumours is sensitive to the adjuvant endocrine treatment which is given for at least 5 years. In contrast, patients with 3 or more residual macrometastases indeed have been shown to have a worse overall survival [26]. This probably creates opportunities to minimize axillary treatment in clinically node-positive HR + HER-patients treated with NET with minimal residual disease in the axilla [26]. For example, the ongoing TAXIS trial is comparing completion ALND to axillary radiotherapy in patients with residual disease in their axillary nodes after NAC or NET, as identified by targeted axillary surgery [27].

To safely minimize axillary treatment in clinically node-positive breast cancer patients, the question is how to restage the axillary lymph nodes after NET. Imaging alone has proven to be insufficient $[28,29]$. In patients treated with NAC, there is an ongoing debate about the preferred technique to stage the axilla, similar to the situation for patients treated with NET. This lack of consensus is also observed in the current study, in which $30.8 \%$ of the patients were treated with an immediate ALND, 24.8\% underwent an SLNB, $25.1 \%$ a MARI procedure, and $19.3 \%$ a combination of MARI and SLNB. The addition of SLNB to MARI resulted in the detection of residual axillary disease in an additional $13 \%$ of the patients. This is 
Table 4

Univariable and multivariable logistic regression analyses for occurrence of axillary pCR.

\begin{tabular}{|c|c|c|c|c|c|c|c|}
\hline & & \multicolumn{3}{|l|}{ Univariable } & \multicolumn{3}{|c|}{ Multivariable } \\
\hline & & Odds ratio & $95 \% \mathrm{CI}$ & $P$ & Odds ratio & $95 \% \mathrm{CI}$ & $P$ \\
\hline \multicolumn{8}{|l|}{ Age years } \\
\hline & $<50$ & Ref. & & 0.16 & Ref. & & 0.17 \\
\hline & $50-75$ & 1.66 & $0.52-5.32$ & & 1.42 & $0.42-4.82$ & \\
\hline & $>75$ & 2.38 & $0.96-5.85$ & & 2.28 & $0.91-5.69$ & \\
\hline \multicolumn{8}{|c|}{ Histologic subtype } \\
\hline & NST & Ref. & & 0.15 & Ref. & & 0.16 \\
\hline & Lobular & 0.42 & $0.13-1.38$ & & 0.41 & $0.12-1.40$ & \\
\hline \multicolumn{8}{|c|}{ Clinical tumor status } \\
\hline & 1 & Ref. & & 0.27 & Ref. & & 0.35 \\
\hline & 2 & 2.05 & $0.85-4.95$ & & 1.87 & $0.75-4.63$ & \\
\hline & $3 / 4$ & 1.37 & $0.60-3.10$ & & 1.18 & $0.51-2.75$ & \\
\hline \multicolumn{8}{|c|}{ Receptor status } \\
\hline & $\mathrm{ER}+\mathrm{PR}+$ HER2- & Ref. & & 0.16 & Ref. & & 0.13 \\
\hline & ER + PR - HER2- & 0.59 & $0.28-1.22$ & & 0.56 & $0.27-1.19$ & \\
\hline \multicolumn{8}{|l|}{ Type NET } \\
\hline & Tamoxifen $(+/-$ GnRH) & Ref. & & 0.18 & Ref. & & 0.10 \\
\hline & Aromatase inhibitor & 0.65 & $0.34-1.22$ & & 0.56 & $0.28-1.12$ & \\
\hline
\end{tabular}

NST $=$ No specific type. ER = estrogen receptor, PR = progesterone receptor, HER2 = human epidermal growth factor receptor 2 , NET = neoadjuvant endocrine therapy.

in line with the reported results of the RISAS study, which reported a false negative rate of only $3.5 \%$ and a negative predictive value of 93.6\% for the combination of MARI and SLNB [30]. The use of the combination of MARI and SLNB after NET seems to be the most balanced procedure to achieve a most accurate restaging with acceptable anticipated morbidity compared to ALND. Nonetheless, the morbidity of the combination of MARI and SLNB compared to MARI alone is under investigation in the Dutch MINIMAX study [31].

Overall, there seems to be no difference between NAC and NET in the occurrence of axillary pCR in patients with HR + HER2tumours with clinically node-positive breast cancer $[15,17]$. However, selection bias could certainly have had an impact in those studies. Extending the duration of NET seems to increase the number of patients with axillary PCR, but the increase is marginal. In order to facilitate more axillary conserving treatment in clinically node-positive HR + HER2-negative breast cancer patients, the addition of other agents such as cyclin-dependent kinase 4/6 (CDK4/6) or phosphatidylinositol 3-kinase (PI3K) inhibitors to standard NET is an ongoing field of research. Results of recent studies regarding achieving pCR are mixed. For example, the use of the PI3K inhibitor taselisib in the LORELEI trial does not significantly increase the pCR rate [32]. Furthermore, the addition of palbociclib to letrozol in the PALLET trial showed a significant reduction in proliferation measured by Ki-67 but showed no significant improvement in clinical response rates after 14 weeks of treatment [33]. Nonetheless, the addition of abemaciclib to adjuvant endocrine therapy in the monarchE study showed a significant invasive disease-free survival benefit to patients with high-risk, HR + HER2-breast cancer patients in the adjuvant setting [34]. At the moment is it unclear whether abemaciclib in the neoadjuvant setting would increase the axillary pCR rate. The ongoing NEOLBC study will give more insights into tailoring neoadjuvant treatment in stage II/III HR + HER2-tumours [35] Thus, there is currently no evidence a CDK4/6 or PI3K inhibitor should be used in the neoadjuvant setting in order to increase the rate of axillary PCR in HR + HER2-tumours in clinically node-positive breast cancer patients.

This study has some limitations, including its retrospective nature and selection bias (regarding selecting patients for NET rather than NAC). Besides, not all patients underwent a completion ALND after a negative SLNB, MARI or the combination of both. All these procedures are known for false negative findings in $2-17 \%$ [36]. The real axillary pCR in the current study would, therefore, be lower when all patients were treated with a completion ALND. Besides, no information was available regarding tumour evaluation during NET, which limited us in providing data on tumour progression during NET. Furthermore, details on follow-up of the patients were not available. Nonetheless, this study is unique regarding its sample size compared to other series (although still limited, probalby causing probably type II errors) and its information on duration of neoadjuvant treatment. It reflects the current standard practice on the use of NET in clinical node-positive HR + HER2-breast cancer patients in the Netherlands, which is still very limited, and provides evidence on the short-term oncologic outcomes of this practice.

\section{CRediT authorship contribution statement}

Robert-Jan Schipper: Conceptualization, Study design, Funding acquisition, Formal analysis, Writing - original draft, Writing review \& editing. Anne de Bruijn: Study design, Funding acquisition, Formal analysis, Writing - original draft, Writing - review \& editing. Adri C. Voogd: Conceptualization, Study design, Funding acquisition, Formal analysis, Writing - original draft, Writing review \& editing. Johanne G. Bloemen: Conceptualization, Writing - review \& editing. Yvonne E. Van Riet: Conceptualization, Writing - review \& editing. Birgit E.P. Vriens: Conceptualization. Marjolein L. Smidt: Conceptualization, Writing - review \& editing. Sabine Siesling: Writing - review \& editing. Maurice J.C. van der Sangen: Conceptualization, Writing - review \& editing. Grard A.P. Nieuwenhuijzen: Conceptualization, Study design, Formal analysis, Writing - review \& editing.

\section{Declaration of competing interest}

We declare to have no conflicts of interest or financial ties to disclose.

\section{References}

[1] van Steenhoven JEC, et al. The changing role of gene-expression profiling in the era of de-escalating adjuvant chemotherapy in early-stage breast cancer. Ann Surg Oncol 2019;26:3495-501.

[2] Kalinsky K. Abstract GS3-00: first results from a phase III randomized clinical trial of standard adjuvant endocrine therapy (ET) $+/$ - chemotherapy (CT) in patients (pts) with 1-3 positive nodes, hormone receptor-positive (HR+) and HER2-negative (HER2-) breast cancer (BC) with recurrence score (RS) $<25$ : SWOG S1007 (RxPonder). SABCS 2020. https://doi.org/10.1158/1538-7445. 
[3] Schreuder K, et al. Use and impact of the 21-gene recurrence score in relation to the clinical risk of developing metastases in early breast cancer patients in The Netherlands. Public Health Genomics 2018;21:85-92.

[4] Cardoso F, et al. 70-Gene signature as an aid to treatment decisions in earlystage breast cancer. N Engl J Med 2016;375:717-29.

[5] Weiss A, et al. Patterns of axillary management in stages 2 and 3 hormone receptor-positive breast cancer by initial treatment approach. Ann Surg Oncol 2019;26:4326-36.

[6] Madigan LI, et al. Neoadjuvant endocrine therapy in locally advanced estrogen or progesterone receptor-positive breast cancer: determining the optimal endocrine agent and treatment duration in postmenopausal women-a literature review and proposed guidelines. Breast Canc Res 2020:22.

[7] Kuehn T, et al. Sentinel-lymph-node biopsy in patients with breast cancer before and after neoadjuvant chemotherapy (SENTINA): a prospective, multicentre cohort study. Lancet Oncol 2013;14:609-18.

[8] Boughey JC, et al. Sentinel lymph node surgery after neoadjuvant chemotherapy in patients with node-positive breast cancer: the ACOSOG Z1071 (Alliance) clinical trial. J Am Med Assoc 2013;310:1455-61.

[9] van der Noordaa MEM, et al. Major reduction in axillary lymph node dissections after neoadjuvant systemic therapy for node-positive breast cancer by combining PET/CT and the MARI procedure. Ann Surg Oncol 2018;25: $1512-20$.

[10] Caudle AS, et al. Improved axillary evaluation following neoadjuvant therapy for patients with node-positive breast cancer using selective evaluation of clipped nodes: implementation of targeted axillary dissection. J Clin Oncol 2016;34:1072-8.

[11] van Nijnatten TJA, et al. A novel less-invasive approach for axillary staging after neoadjuvant chemotherapy in patients with axillary node-positive breast cancer by combining radioactive iodine seed localization in the axilla with the sentinel node procedure (RISAS): a Dutch prospective multicenter validation study. Clin Breast Canc 2017;17:399-402.

[12] Samiei S, et al. Correlation between pathologic complete response in the breast and absence of axillary lymph node metastases after neoadjuvant systemic therapy. Ann Surg 2020;271:574-80.

[13] Petruolo OA, et al. Standard pathologic features can Be used to identify a subset of estrogen receptor-positive, HER2 negative patients likely to benefit from neoadjuvant chemotherapy. Ann Surg Oncol 2017:24:2556-62.

[14] von Minckwitz G, et al. Definition and impact of pathologic complete response on prognosis after neoadjuvant chemotherapy in various intrinsic breast cancer subtypes. J Clin Oncol 2012;30:1796-804

[15] Stafford A, et al. Axillary response in patients undergoing neoadjuvant endocrine treatment for node-positive breast cancer: systematic literature review and NCDB analysis. Ann Surg Oncol 2020;27:4669-77.

[16] Dutch breast cancer guideline; 2020. City.

[17] Montagna G, et al. How effective is neoadjuvant endocrine therapy (NET) in downstaging the axilla and achieving breast-conserving surgery? Ann Surg Oncol 2020;27:4702-10.

[18] Weigelt B, et al. Refinement of breast cancer classification by molecular characterization of histological special types. J Pathol 2008;216:141-50.

[19] Truin W, et al. Estrogen and progesterone receptor expression levels do not differ between lobular and ductal carcinoma in patients with hormone receptor-positive tumors. Breast Canc Res Treat 2017;164:133-8.

[20] Thornton MJ, et al. Neoadjuvant endocrine therapy versus neoadjuvant chemotherapy in node-positive invasive lobular carcinoma. Ann Surg Oncol 2019;26:3166-77.
[21] Christgen M, et al. Differential impact of prognostic parameters in hormone receptor-positive lobular breast cancer. Cancer 2020;126:4847-58.

[22] Conlon $\mathrm{N}$, et al. Is there a role for oncotype dx testing in invasive lobular carcinoma? Breast J 2015;21:514-9.

[23] Lesnikoski B-A, et al. Molecular profiles and treatment recommendations for invasive lobular carcinoma in a real-world prospective breast cancer registry. J Clin Oncol 2020;38. e19291-e.

[24] Mamounas EP, et al. NRG Oncology/NSABP B-51/RTOG 1304: phase III trial to determine if chest wall and regional nodal radiotherapy (CWRNRT) post mastectomy (Mx) or the addition of RNRT to whole breast RT post breastconserving surgery (BCS) reduces invasive breast cancer recurrence-free interval (IBCR-FI) in patients (pts) with pathologically positive axillary (PPAx) nodes who are ypNO after neoadjuvant chemotherapy (NC). I Clin Oncol 2019;37.

[25] Cortazar P, et al. Pathological complete response and long-term clinical benefit in breast cancer: the CTNeoBC pooled analysis. Lancet 2014;384: $164-72$.

[26] Kantor O, et al. Prognostic significance of residual nodal disease after neoadjuvant endocrine therapy for hormone receptor-positive breast cancer. Npj Breast Cancer 2020:6.

[27] Henke G, et al. Tailored axillary surgery with or without axillary lymph node dissection followed by radiotherapy in patients with clinically node-positive breast cancer (TAXIS): study protocol for a multicenter, randomized phaseIII trial. Trials 2018;19:667.

[28] Schipper RJ, et al. Noninvasive nodal restaging in clinically node positive breast cancer patients after neoadjuvant systemic therapy: a systematic review. Eur J Radiol 2014.

[29] Samiei S, et al. Diagnostic performance of noninvasive imaging for assessment of axillary response after neoadjuvant systemic therapy in clinically nodepositive breast cancer: a systematic review and meta-analysis. Ann Surg 2021;273:694-700.

[30] Simons J, et al. Radioactive Iodine Seed placement in the Axilla with Sentinel lymph node biopsy after neoadjuvant chemotherapy in breast cancer: results of the prospective multicenter RISAS trial. Canc Res 2021;81. https://doi.org/ 10.1158/1538-7445. GS1-10-GS1.

[31] Smidt ML. Minimal invasive axillary staging and treatment after neoadjuvant systemic therapy in node positive breast cancer (MINIMAX). https:/ clinicaltrials.gov/ct2/show/NCT04486495.

[32] Saura C, et al. Neoadjuvant letrozole plus taselisib versus letrozole plus placebo in postmenopausal women with oestrogen receptor-positive, HER2negative, early-stage breast cancer (LORELEI): a multicentre, randomised, double-blind, placebo-controlled, phase 2 trial. Lancet Oncol 2019;20: $1226-38$.

[33] Johnston S, et al. Randomized phase II study evaluating palbociclib in addition to letrozole as neoadjuvant therapy in estrogen receptor-positive early breast cancer: PALLET trial. J Clin Oncol 2019;37:178-89.

[34] Johnston SRD, et al. Abemaciclib combined with endocrine therapy for the adjuvant treatment of HR+, HER2-, node-positive, high-risk, early breast cancer (monarchE). J Clin Oncol 2020;38:3987-98.

[35] Kroep JR. Tailoring NEOadjuvant therapy in hormone receptor positive, HER2 negative, luminal breast cancer. (NEOLBC). https://clinicaltrials.gov/ct2/show/ NCT03283384.

[36] Simons JM, et al. Diagnostic accuracy of different surgical procedures for axillary staging after neoadjuvant systemic therapy in node-positive breast cancer: a systematic review and meta-analysis. Ann Surg 2019;269:432-42. 